\title{
FREQUÊNCIA FUNDAMENTAL DA VOZ DE CRIANÇAS
}

\author{
Fundamental frequency of children's voice
}

\author{
Júnia Novaes Braga ${ }^{(1)}$, Domingos Sávio Ferreira de Oliveira ${ }^{(2)}$, Tania Maria Marinho Sampaio(3)
}

\begin{abstract}
RESUMO
Objetivo: determinar a frequência fundamental (Fo) da voz de 50 meninos e 50 meninas, nascidos e residentes em Belo Horizonte na faixa etária de seis a oito anos. Métodos: foram selecionadas crianças de ambos os sexos da cidade de Belo Horizonte. O protocolo de gravação das vozes foi realizado a partir da digitalização da vogal sustentada $[\varepsilon]$ em tom e intensidade adequada, com duração de três segundos, e em ambiente silencioso. Utilizou-se o programa de Análise de Voz Voxmetria ${ }^{\circledR}$. Resultados: os achados mostraram a média total da frequência fundamental em $249,71 \mathrm{~Hz}$, a diminuição significante nos valores de $\mathrm{F}_{0}$ com o aumento da idade nos dois sexos, e diferenças significantes de $F_{0}$ entre os sexos comparando os sexos e as idades das crianças. Conclusão: a frequência fundamental da voz de crianças na faixa etária estudada apresentou média de $249,71 \mathrm{~Hz}$.
\end{abstract}

DESCRITORES: Criança; Laringe; Qualidade da Voz; Acústica da Fala

\section{INTRODUÇÃO}

A voz é uma das formas de comunicação com o exterior, sendo exclusiva dos seres humanos. Possui características próprias que variam de acordo com o sexo, pessoa e faixa etária, além de refletir o estado e o comportamento laríngeo, caracterizando o que se chama de qualidade vocal ${ }^{1-4}$.

Pouco se conhece a respeito da produção da voz na infância, etapa esta em que inúmeras transformações ocorrem na laringe de ordem anatômica, morfológica e histológica. Este órgão sofre diversas modificações principalmente nos primeiros anos de vida. Por isso torna-se instigante e desafiador analisar a voz da criança, a cada ano de vida, pois este corresponde a diversas fases de desenvolvimento ${ }^{1,4-7}$.

Para aprofundamento dos estudos da voz, fazse necessário o entendimento da fisiologia e da acústica fonatória normais e, para descrever as características de uma voz, vários procedimentos são utilizados, como as avaliações perceptivo-audi-

(1) Fonoaudióloga; Clínica Cliserv, Belo Horizonte, MG; Mestre em Fonoaudiologia pela Universidade Veiga de Almeida.

(2) Fonoaudiólogo; Diretor-Presidente Clínica da Voz, Niterói, RJ; Professor Adjunto da Universidade Veiga de Almeida, UVA, Rio de Janeiro, RJ e da Universidade do Rio de Janeiro, Rio de Janeiro, RJ; Doutor em Lingüística/Fonética Experimental pela Universidade Federal Fluminense.

(3) Filósofa; Professora Adjunto da Universidade Veiga de Almeida, UVA, Rio de Janeiro, RJ; Doutora em Filosofia pela Universidade Federal Fluminense. tiva e acústica. A avaliação perceptivo-auditiva é subjetiva, considerada soberana e muito utilizada na prática clínica ${ }^{3,4,8-10}$. A avaliação acústica não é uma realidade na clínica fonoaudiológica, mas vem contribuindo bastante para a determinação de parâmetros de normalidade por ser uma avaliação objetiva, que possibilita o armazenamento de dados para posteriores comparações ${ }^{3,11-14}$.

Dentre os vários parâmetros vocais acústicos que podem ser obtidos através de programas ou equipamentos específicos, a frequência fundamental (Fo) se destaca como um dos mais importantes. Corresponde ao número de ciclos que as pregas vocais fazem em um segundo, e sua extração tem se mostrado o parâmetro mais resistente aos diversos sistemas de análise acústica. Os fatores de determinação da frequência fundamental e sua variação em extensão vocal são representados pelo comprimento natural das pregas vocais, pelo alongamento, pela massa em vibração e pela tensão envolvida ${ }^{11,15-18}$. A análise dos parâmetros vocais de frequência fundamental é de grande importância para o estudo do desenvolvimento da voz em função da idade ${ }^{4,11,15,16}$.

O conhecimento da voz na infância ainda é escasso. Com a utilização do laboratório de voz na extração da frequência fundamental das crianças com vozes normais pode-se estabelecer um padrão de voz específico à criança, o que auxiliaria na normatização de um modelo de comportamento vocal adequado à infância ${ }^{15-17,19}$. 
O objetivo do presente trabalho foi determinar a frequência fundamental $\left(F_{0}\right)$ da voz de 50 meninos e 50 meninas nascidos e residentes em Belo Horizonte, na faixa etária de seis a oito anos.

\section{MÉTODOS}

Participaram deste estudo 100 crianças, 50 meninos e 50 meninas com idade variando de seis a oito anos, estudantes do ensino fundamental da Escola Estadual Presidente Antônio Carlos, localizada na região sul, nascidos e residentes na cidade de Belo Horizonte, Minas Gerais.

Da amostra inicial de 150 crianças foram excluídas 50 , sendo 30 por apresentarem um ou mais dos critérios de exclusão durante a avaliação (Figura 1) e 20 pela disfonia em grau moderado, segundo a escala RASAT. As crianças que apresentaram grau leve de rouquidão e soprosidade foram incluídas por se considerar tais características comuns em crianças ${ }^{7,10,13}$.

O procedimento consistiu na gravação do nome do participante, idade, vogal sustentada $[\varepsilon]$ três vezes consecutivas, com uma pequena pausa entre elas. Em seguida, foi gravada a fala encadeada a partir da contagem dos números de zero a dez. Orientou-se que as crianças emitissem os referidos sons em frequência, intensidade e qualidade vocal mais natural e confortável possível.

As amostras vocais foram capturadas utilizando o programa Voxmetria, microfone unidirecional da marca Shure SM 58, fixado a um tripé ajustado à altura da criança, formando um ângulo de aproximadamente 45 graus e com $5 \mathrm{~cm}$ de distância da boca. As gravações das amostras vocais foram capturadas diretamente no computador em ambiente silente. Com o material obtido foram realizadas duas análises: perceptivo-auditiva e acústica.

A análise perceptivo-auditiva foi realizada por 3 fonoaudiólogas experientes utilizando a escala RASAT, com consenso entre os juízes. Para a análise acústica foi utilizada emissão vocal de três segundos, eliminando-se o início e o fim da emissão, devido às instabilidades inerentes a estes períodos.

Este trabalho foi aprovado pelo Comitê de Ética em Pesquisa da Universidade Veiga de Almeida sob o número resolução 96/08.

Nome:

Sexo: ( ) Masculino ( ) Feminino

Data de Nascimento: 1__

Série:

Mora em Belo Horizonte? ( ) sim ( ) não

( ) menos de 5 anos ( ) mais de 5 anos

Local de nascimento:

A criança apresenta ou já apresentou alguma alteração na voz? （） sim （）não

Descreva-a

A criança fica rouca frequentemente? ( ) sim ( ) não

A criança apresenta distúrbio auditivo? ( ) sim ( ) não

Apresenta frequentemente:
( ) laringite
( ) bronquite
( ) rinite
( ) sinusite
( ) asma
( ) otite
( ) faringite
( ) amigdalite
( ) outros
A criança está resfriada ou gripada? ( ) sim ( ) não

IC: anos meses 
A análise descritiva para a frequência fundamental foi realizada por meio de medidas de tendência central (média e mediana) e de variabilidade (desvio padrão, valores mínimo e máximo). Esses resultados são apresentados para a amostra total, para cada sexo, para cada idade (6, 7 ou 8 anos) e também para as interações entre o sexo e a idade. A comparação dos grupos é feita graficamente através de boxplots.

A normalidade da distribuição da frequência fundamental foi verificada através do teste de ShapiroWilk com um nível de significância de $5 \%$. Como não se observou uma distribuição normal na frequência fundamental, utilizou-se o intervalo de confiança pelo método dos percentis. O intervalo pelo método de Gauss é apresentado apenas para uma possível comparação com outros resultados descritos na literatura. Para verificar se existe diferença entre as observações nos grupos com 6, 7 ou 8 anos de idade, em cada sexo, foi utilizado o teste não-paramétrico de Kruskal Wallis.

\section{RESULTADOS}

A Tabela 1 apresenta as estatísticas descritivas para a frequência fundamental $(\mathrm{Hz})$ para a amostra total, e de acordo com o sexo e a idade das crianças avaliadas.

Realizou-se comparação de $F_{0}$ por sexo, feita através do teste não-paramétrico de Kruskal-Wallis (Tabela 2). Em ambos os sexos, o resultado foi significante (valor $p<0,05$ ) e assim observou-se que há diferença significante na frequência fundamental aos seis, sete ou oito anos de idade, com um nível de significância de $5 \%$.

Ao se comparar $F_{0}$ entre as idades estudadas, através do teste não-paramétrico de MannWhitney, observou-se diferenças consideradas significantes na frequência fundamental aos seis, sete ou oito anos de idade, quando comparadas duas a duas em cada sexo, com um nível de significância de $5 \%$ (Tabela 3).

Tabela 1 - Estatísticas descritivas para a Frequência Fundamental

\begin{tabular}{llcccccc}
\hline Sexo & Idade & N & Média & $\begin{array}{c}\text { Desvio } \\
\text { Padrão }\end{array}$ & Mínimo & Mediana & Máximo \\
\hline Feminino & 6 anos & 16 & 255,85 & 4,08 & 248,63 & 255,52 & 262,06 \\
& 7 anos & 17 & 246,01 & 3,39 & 241,42 & 245,50 & 253,80 \\
& 8 anos & 17 & 231,08 & 2,86 & 226,52 & 230,15 & 235,45 \\
\hline Masculino & 6 anos & 16 & 271,46 & 3,31 & 267,14 & 271,79 & 277,62 \\
& 7 anos & 17 & 255,08 & 4,43 & 247,29 & 254,48 & 261,71 \\
& 8 anos & 17 & 240,45 & 3,00 & 236,15 & 240,62 & 245,25 \\
\hline Total Feminino & 50 & 244,08 & 3,43 & 226,52 & 245,41 & 262,06 \\
Total Masculino & 50 & 255,35 & 3,59 & 236,15 & 253,99 & 277,62 \\
\hline Total 6 anos & 32 & 263,65 & 8,73 & 248,63 & 264,60 & 277,62 \\
Total 7 anos & 34 & 250,55 & 6,02 & 241,42 & 250,23 & 261,71 \\
Total 8 anos & 34 & 235,77 & 5,56 & 226,52 & 235,80 & 245,25 \\
\hline Total Geral & 100 & 249,71 & 3,51 & 226,52 & 248,95 & 277,62 \\
\hline
\end{tabular}

Tabela 2 - Teste de Kruskal-Wallis para comparação da $F_{0}$ por sexo

\begin{tabular}{lccc}
\hline Sexo & $\begin{array}{c}\text { Estatística } \\
\text { de teste }\end{array}$ & GI & Valor $\mathbf{p}$ \\
\hline Feminino & 41,923 & 2 & 0,000 \\
Masculino & 43,562 & 2 & 0,000 \\
\hline
\end{tabular}

Tabela 3 - Teste de Mann-Whitney para comparação da $F_{0}$ aos 6, 7 e 8 anos

\begin{tabular}{llc}
\hline Sexo & Comparação & Valor $\mathbf{p}$ \\
\hline Feminino & 6 anos $\times 7$ anos & 0,000 \\
& 6 anos $\times 8$ anos & 0,000 \\
& 7 anos $\times 8$ anos & 0,000 \\
\hline Masculino & 6 anos $\times 7$ anos & 0,000 \\
& 6 anos $\times 8$ anos & 0,000 \\
& 7 anos $\times 8$ anos & 0,000 \\
\hline
\end{tabular}


Comparando ainda Fo entre meninos e meninas, independente da idade, observou-se que há diferenças significantes $(p=0,000)$ entre os resultados, com um nível de significância de 5\%.

Os resultados dos intervalos obtidos por cada um dos dois métodos para determinação de limites de normalidade são apresentados nas Tabelas 4 e 5. Qualquer generalização desses resultados deve ser feita com cuidado, pois há um pequeno número de indivíduos para se determinar uma faixa de normalidade para a população.

Tabela 4 - Intervalo de $95 \%$ de confiança para a Frequência Fundamental de acordo com o Sexo e a Idade - Método de Gauss

\begin{tabular}{lccccc}
\hline Sexo & N & Média & $\begin{array}{c}\text { Desvio } \\
\text { Padrão }\end{array}$ & IC 95\% \\
\hline Feminino & Total & 50 & 244,08 & 3,43 & 222,$91 ; 265,25$ \\
& 6 anos & 16 & 255,85 & 4,08 & 247,$85 ; 263,85$ \\
& 7 anos & 17 & 246,01 & 3,39 & 239,$37 ; 252,65$ \\
& 8 anos & 17 & 231,08 & 2,86 & 225,$47 ; 236,69$ \\
\hline Masculino & Total & 50 & 255,35 & 3,59 & 229,$46 ; 281,24$ \\
& 6 anos & 16 & 271,46 & 3,31 & 264,$97 ; 277,95$ \\
& 7 anos & 17 & 255,08 & 4,43 & 246,$40 ; 263,76$ \\
& 8 anos & 17 & 240,45 & 3,00 & 234,$57 ; 246,33$ \\
\hline
\end{tabular}

Tabela 5 - Percentis de 2,5\% e 97,5\% para a Frequência Fundamental de acordo com o Sexo e a Idade Método dos Percentis

\begin{tabular}{lccccc}
\hline Sexo & Idade (anos) & $\mathbf{N}$ & Percentil 2,5 & Percentil 97,5 & IC 95\% \\
\hline Feminino & 6 anos & $\mathrm{N}=16$ & 248,63 & 262,06 & 248,$63 ; 262,06$ \\
& 7 anos & $\mathrm{N}=17$ & 241,42 & 253,80 & 241,$42 ; 253,80$ \\
& 8 anos & $\mathrm{N}=17$ & 226,52 & 235,45 & 226,$52 ; 235,45$ \\
& Total & $\mathrm{N}=50$ & 226,69 & 261,86 & 226,$69 ; 261,86$ \\
\hline Masculino & 6 anos & $\mathrm{N}=16$ & 267,14 & 277,62 & 267,$14 ; 277,62$ \\
& 7 anos & $\mathrm{N}=17$ & 247,29 & 261,71 & 247,$29 ; 261,71$ \\
& 8 anos & $\mathrm{N}=17$ & 236,15 & 245,25 & 236,$15 ; 245,25$ \\
& Total & $\mathrm{N}=50$ & 236,18 & 276,94 & 236,$18 ; 276,94$ \\
\hline
\end{tabular}

\section{DISCUSSÃO}

A análise acústica trata-se de um método não invasivo que propõe a avaliar vozes normais e disfônicas. Porém, seus resultados ainda não podem ser generalizados devido à falta de normatização das medidas ${ }^{2,5,13-15}$. Dentre os estudos realizados, na tentativa de estabelecer a normatização de medidas acústicas para vozes infantis, podem-se destacar alguns ${ }^{9,15,19-21}$.

No presente estudo, a média da frequência fundamental de $249,71 \mathrm{~Hz}$ das 100 crianças dos sexos feminino e masculino de 6 a 8 anos de Belo Horizonte, coincide com os dados disponíveis na literatura estudada. Num estudo realizado em 1997 com crianças de ambos os sexos, de seis a 10 anos, a média da $F_{0}$ encontrada foi de $250,05 \mathrm{~Hz}{ }^{21}$. Outros autores encontraram o valor de referência de normalidade acima de $250 \mathrm{~Hz}^{12,15}$. Em uma pesquisa ${ }^{10}$ verificou média de $F_{0}$ igual a $237 \mathrm{~Hz}$, sendo o valor inferior a esta pesquisa e àqueles relatados anteriormente.

A faixa etária analisada no trabalho foi escolhida para que não houvesse qualquer interferência nos parâmetros vocais causados pela muda vocal e por representar um período que não há diferença significante na laringe da criança entre os sexos $1,6,7,13$.

A frequência fundamental é uma das medidas mais importantes da análise acústica e tem relação direta com o comprimento, tensão, rigidez e massa 
das pregas vocais e estas com a pressão subglótica ${ }^{2,4,16,17}$. Neste estudo, as médias da frequência fundamental encontradas nas faixas etárias de seis, sete e oito anos foram de $263,65 \mathrm{~Hz}, 250,55 \mathrm{~Hz}$ e $235,77 \mathrm{~Hz}$ respectivamente, percebendo uma diminuição significativa nos valores. Este achado está de acordo com a literatura, pois a maioria dos autores concorda que quanto maior a idade, menor o valor na média da frequência fundamental para ambos os sexos (Figura 2), pois à medida que a criança cresce as estruturas do trato vocal também crescem e, consequentemente, as pregas vocais aumentam seu comprimento e massa, diminuindo a frequência fundamental $1,10,21,22$. Essa diminuição da $F_{0}$ é bem perceptível se observar as extremidades das faixas-etárias de oito anos e de seis anos $5,17,21,22$ (Tabela 1). As justificativas mais relatadas para a diminuição da $F_{0}$ são as mudanças anatômicas e fisiológicas nas próprias estruturas laríngeas, decorrente do desenvolvimento normal, e a maturação do sistema nervoso, possibilitando maior controle laríngeo. Este fato justifica em parte as observações deste estudo.

Algo que chama atenção é a comparação das médias de $F_{0}$ em ambos os sexos de acordo com a idade e entre os sexos independente da idade (Figuras 3 e 4). Neste estudo, observa-se diferença gradativa com queda da frequência fundamental aos seis, sete e oito anos nos dois sexos, e diferenças significantes de $\mathrm{F}_{0}$ comparando um com outro. Encontra-se na literatura consultada apenas um estudo ${ }^{23}$ concordando com a significância quanto à idade e ao sexo.

A média da frequência fundamental das crianças do sexo masculino encontrada ${ }^{10}$ foi superior aos valores da média da frequência fundamental das

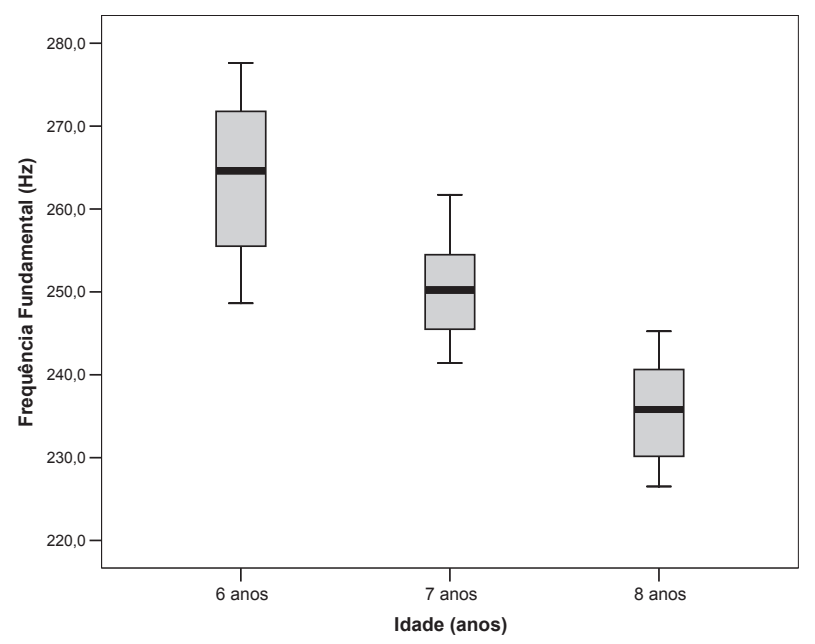

Figura 2 - Boxplots para a Frequência Fundamental de acordo com a idade crianças de sexo feminino, concordando com os achados: nesse estudo citado anteriormente o valor encontrado da média de frequência fundamental foi de $241,96 \mathrm{~Hz}$ no sexo masculino e de $233,04 \mathrm{~Hz}$ no feminino, apresentando uma diferença de $8,92 \mathrm{~Hz}$ no valor da média de $F_{0}$. Outros autores não compartilham deste achado, pois não encontraram diferenças significantes da média da frequência fundamental entre meninos e meninas ${ }^{22}$.

A Figura 4 é uma amostra comparativa da frequência fundamental de acordo com a idade e com o sexo. Os resultados revelam diferenças bastante significantes quando confrontados o sexo e a idade juntos. Observa-se, por exemplo, que o menino apresenta uma frequência fundamental mais elevada se comparada à menina. A queda em ambos é esperada, regular e equidistante, sendo um pouco mais evidente no menino na idade de seis a oito anos (Tabela 1). Discordando dos achados ${ }^{22}$ não foram encontradas diferenças significativas de $F_{0}$ entre os sexos ou comparando os sexos e as idades das crianças estudadas.

Numa proposta de investigar características acústicas da voz na criança quase pré-adolescente em relação ao sexo e à idade ${ }^{23}$ revelaram que as diferenças de frequência fundamental entre crianças do sexo masculino e feminino surgem na idade de 7 anos, e essa frequência fundamental diminui significantemente apenas nas crianças do sexo masculino, entre a idade de cinco a 10 anos. Neste estudo observa-se esta diminuição em ambos os sexos. Estes achados são curiosos ao comparar à literatura, pois se sabe que o desenvolvimento anatômico da laringe na infância é semelhante até a pré-adolescência, o que influencia a qualidade da voz. Em geral, não há diferenças significativas entre

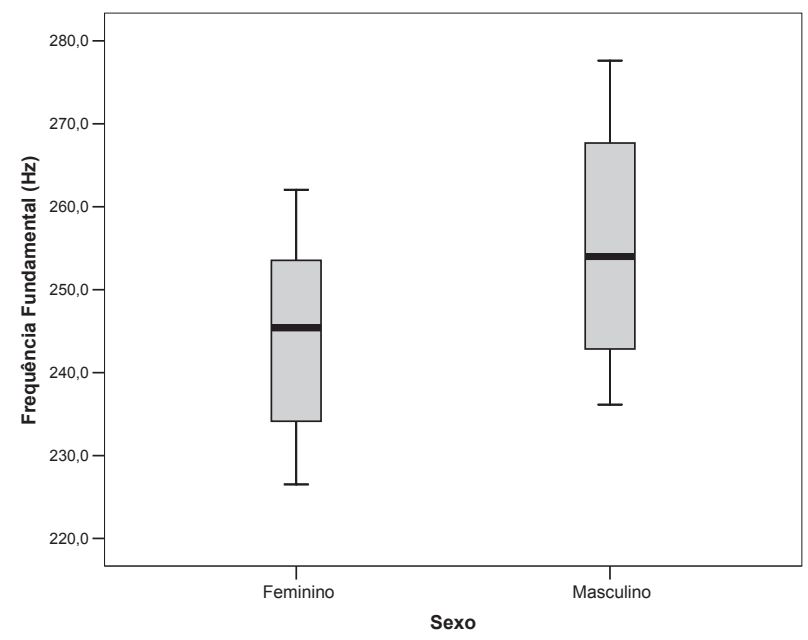

Figura 3 - Boxplots para a Frequência Fundamental de acordo com o sexo 


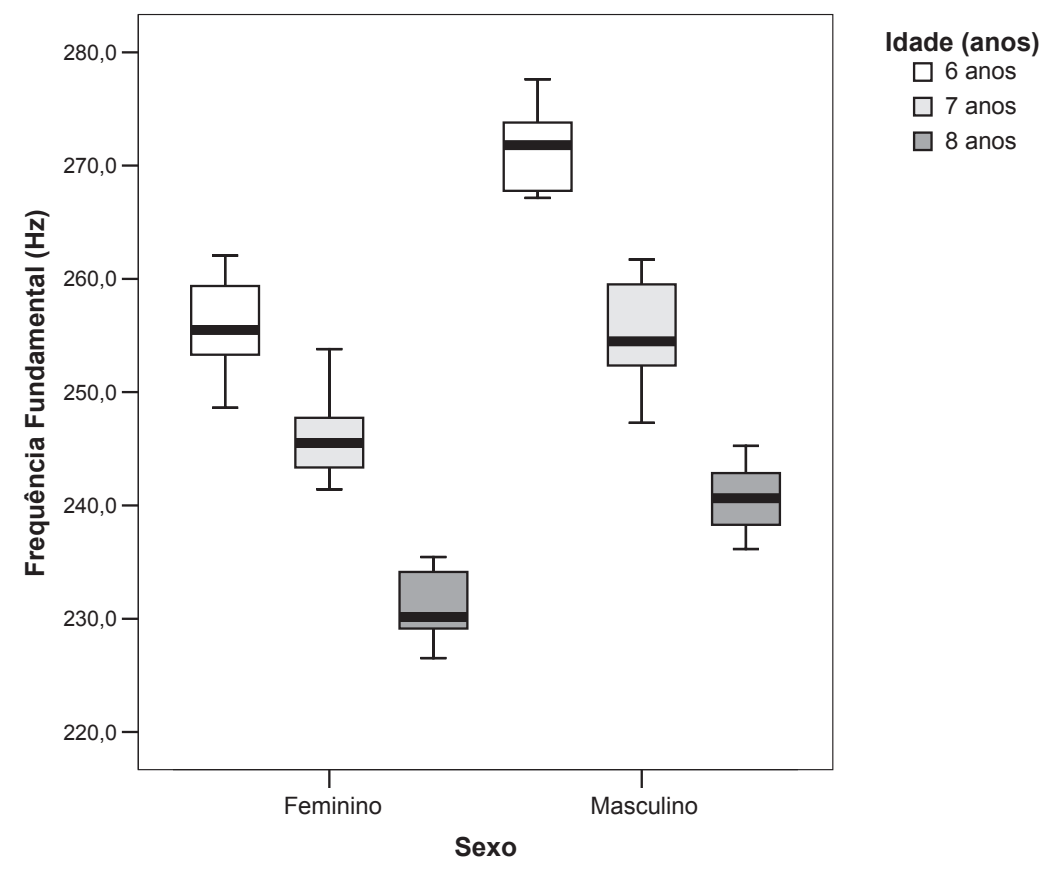

Figura 4 - Boxplots para a Frequência Fundamental de acordo com a idade e com o sexo

as vozes de meninos e meninas, isto é, não se percebe diferenças de tonalidade $9,13,17,22$.

De acordo com a literatura, isso ocorre porque até a puberdade a laringe apresenta as mesmas dimensões tanto no menino quanto na menina ${ }^{1,4,13}$. Por isso nessa faixa etária nem sempre é possível diferenciar o sexo de uma criança ouvindo apenas a voz, sobretudo em relação aos meninos. A diferença só será marcante e bem definida a partir da puberdade.

No estudo realizado, encontrou-se valores distintos de $\mathrm{F}_{0}$ nos dois sexos. Este achado pode ser explicado com base em fatos orgânicos, devido ao ritmo diferente de amadurecimento sexual para meninos e meninas ou comportamentais, pois meninos utilizam atividades lúdicas e desportivas bastante energéticas que geram hipertensão cervical provocando, como consequência, contração muscular excessiva dos músculos da fonação. Esta contração influencia a qualidade vocal caracterizando a voz com um pitch mais agudo.

Não se encontram na literatura estudos que expliquem o fato da $F_{0}$ ser mais elevada no menino. Considerando que a amostra deste estudo é pequena, este fato requer, naturalmente, maiores investigações. A velocidade com que as mudanças orgânicas e fisiológicas ocorrem nas crianças suscita novos conhecimentos e pesquisas sobre 0 desenvolvimento da voz infantil, pois as mudanças que influenciarão a voz e o crescimento da laringe ocorrem de modo desigual e desordenado, sobretudo no menino, acarretando características vocais bastante específicas, influenciando a frequência fundamental. Assim, este estudo revela achados interessantes que instiga a continuar pesquisando o universo infantil.

A amostra pesquisada é uma contribuição efetiva para a determinação da frequência fundamental da criança brasileira, em especial, de Belo Horizonte e este estudo é um estímulo para novas pesquisas e para maior entendimento da voz na infância, embora essa amostra seja referente a 100 crianças na faixa etária de seis a oito anos.

\section{CONCLUSÃO}

A média da frequência fundamental das 100 crianças dos sexos feminino e masculino de seis a oito anos nascidas e residentes em Belo Horizonte é de $249,71 \mathrm{~Hz}$, e esta diminui significantemente à medida em que aumenta a idade.

No sexo masculino, aos seis anos, a média da frequência fundamental apresenta valor em $\mathrm{Hz}$ superior à média encontrada para meninas, e diminui significantemente nas idades de sete e oito anos. No sexo feminino, observou-se a queda da média da $F_{0}$, porém, menos significante se comparada ao menino.

Os resultados deste estudo reúnem dados relevantes para trabalhar a voz infantil de maneira mais profunda e segura, contribuindo para a padronização da frequência fundamental desta população. 


\section{ABSTRACT}

Purpose: to determine the fundamental frequency (Fo) for the voice of 50 boys and 50 girls born and living in Belo Horizonte, whose ages range from 6 to 8 years. Methods: both genders were chosen from Belo Horizonte city. The process of voice recording was done by using digital sustained vowel $[\varepsilon]$ within proper tone and intensity, lasting three seconds, in a silent surrounding. We used Voice Analysis Voxmetria ${ }^{\circledR}$ software, best indicated to extract fundamental frequency $\left(F_{0}\right)$, main objective of this research. Results: the findings showed the average of fundamental frequency being equal to $249.71 \mathrm{~Hz}$, a meaningful decrease in Fo values as the age increases in both genders, and greater differences in $F_{0}$ between genders. Conclusion: the average fundamental frequency of children's voice between 6 to 8 -year-old is $249.71 \mathrm{~Hz}$.

KEYWORDS: Child; Larynx; Voice Quality; Speech Acoustics

\section{REFERÊNCIAS}

1. Lima LRS, Campelo VES, Nita LM, Imamura R, Tsuji DH, Sennes LU. Peculiaridades da laringe infantil. Vox Brasilis. 2005; 11(13):12-4.

2. Melo ECM, Lemos M, Ximenes JA, Sennes LU, Saldiva PHN, Tsuji DH. Distribution of collagen in the lamina propria of the human vocal fold. Laryngoscope. 2003; 113(12):2187-91.

3. Bele IV. Reliability in perceptual analysis of voice quality. J Voice. 2005; 19(4):555-73.

4. Hirano S, Bless DM, del Rio AM, Connor NP, Ford CN. Therapeutic potential of growth factors for aging voice. Laryngoscope. 2004; 114(12):2161-7.

5. Ximenes Filho JA, Melo ECM, Carneiro CG, Tsuji DH, Sennes LU. Correlação entre a altura e as dimensões das pregas vocais. Rev Bras Otorrinolaringol. 2003; 69(3):371-4.

6. Santos MAO, Moura JMP, Duprat AC, Costa $\mathrm{HO}$, Azevedo BB. A interferência da muda vocal nas lesões estruturais das pregas vocais. Rev Bras Otorrinolaringol. 2007; 73(2):226-30.

7. Martins RHG, Trindade SHK. A criança disfônica: diagnóstico, tratamento e evolução clínica. Rev Bras Otorrinolaringol. 2003; 69(6):801-6.

8. Pinho S, Pontes P. Escala de avaliação perceptiva da fonte glótica: RASAT. Vox Brasilis. 2002; 8(3):11-3.

9. Sader RM, Hanayama EM. Considerações teóricas sobre a abordagem acústica da voz infantil. Rev CEFAC. 2004; 6(3):312-8.

10. Vanzella TH. Normatização dos parâmetros acústicos vocais em crianças em idade Escolar. [dissertação] Ribeirão Preto (SP): Universidade de São Paulo; 2006.

11. Araújo SA, Grellet M, Pereira JC. Normatização de medidas acústicas da voz normal. Rev Bras Otorrinolaringol. 2002; 68(4):540-4.
12. Honorof DN, Whalen DH. Perception of pitch location within a speaker's $F_{0}$ range. J Acoust Soc Am. 2005; 117(4 Pt1):2193-200.

13. Freitas MR, Weckx LLM, Pontes PA. Disfonia na infância. Rev Bras Otorrinolaringol. 2000; 66(3):257-64.

14. Nemr K, Amar A, Abrahão M, Leite GCA, Kohler J, Santos A, Correa LAC. Análise comparativa entre avaliação fonoaudiológica perceptivo-auditiva, análise acústica e laringoscopias indiretas para avaliação vocal em população com queixa vocal. Rev Bras Otorrinolaringol. 2005; 71(1):13-7.

15. Jotz GP, Cervantes O, Settani FAP, Angelis EC. Acoustic measures for the detection of hoarseness in children. Arq Int Otorrinolaringol. 2006; 10(1):14-20.

16. Dejonckere $\mathrm{PH}$, Wieneke $\mathrm{GH}$, Bloemenkamp $\mathrm{D}$, Lebacq J. $\mathrm{F}_{0}$ : perturbation and $\mathrm{FO} /$ loudness dynamics in voices of normal children, with and without education in singing. Int $\mathrm{J}$ Pediatr Otorhinolaryngol. 1996; 35(2):107-15.

17. Marinho JS, Borges AN, Ramos SM, Silvestre $\mathrm{KR}$. Frequência fundamental de crianças de 5-6 anos antes e depois do intervalo escolar. Estudos Vida e Saúde. 2003; 30(7):1763-78.

18. McAllister A, Sederholm E, Sundberg J. Perceptual and acoustic analysis of vocal registers in 10-year-old children. Logoped Phoniatr Vocol. 2000; 25(2):63-71.

19. Campisi P, Tewfik TL, Manoukian J, Schloss MD, Pelland-Blais E, Sadeghi N. Computerassisted voice analysis: establishing a pediatric database. Arch Otolaryngol Head Neck Surg. 2002; 128(2):156-60.

20. Hufnagle J. Acoustic analysis of fundamental frequencies of voices of children with and without vocal nodules. Percept Mot Skills. 1982; 55(2):427-32. 
21. Steffen N, Moschetti MB, Wolff C, Dossin L, Oussale M, Keil DT. Frequência fundamental de 131 crianças de 06 a 10 anos estudantes de Porto Alegre relacionada com idade, peso, estatura física e superfície corporal. Rev Soc Bras Fonoaudiol. 1997; 1:22-8.

22. Navas DM. Análise computadorizada de freqüência fundamental e suas variaçöes em altura (jitter) e intensidade (shimmer) de vozes de crianças da cidade de Säo Paulo. Pró-Fono. 1989; 1(1):17-22.

23. Fairbanks G. An acoustical comparison of vocal pitch in seven- and eight-year-old children. Child Dev. 1950; 21(2):121-9.

24. Hasek CS, Singh S, Murry T. Acoustic attributes of preadolescent voices. J Acoust Soc Am. 1980; 68(5):1262-5.

RECEBIDO EM: 15/03/2008

ACEITO EM: 03/11/2008

Endereço para correspondência:

Junia Novaes Braga

Rua Correias, 365 ap. 600

Belo Horizonte - MG

CEP: 30315-340

E-mail: jbraga@terra.com.br 